\title{
Boron Nitride Nanostructured: Synthesis, Characterization and Potential Use in Cosmetics
}

\author{
Liliam Márcia Silva Ansaloni, Edésia Martins Barros de Sousa*
}

Nuclear Technology Development Center, Universidade Federal De Minas Gerais, Belo Horizonte, Brazil.

Email: *sousaem@cdtn.br

Received October $19^{\text {th }}, 2012$; revised November $21^{\text {st }}, 2012$; accepted December $12^{\text {th }}, 2012$

\begin{abstract}
The hexagonal boron nitride has been well investigated. Its layered structure is similar to that of graphite. In the manufacture of cosmetics, this structure is intended to improve the appearance, sensory aspect and makes it easy to mix excipients contained in the formulation in the mixture process. In cosmetology, the efficiency and the penetration of active substances into skin are known to be directly related to the particle size. However, only recently their nanostructured properties have been explored. In this work, the synthesis of hexagonal boron nitride in the nanoscale, its composition, structure, morphology and potential for future application in sunscreens are being investigated. The boron nitride was synthesized from boric acid and melamine to a heat treatment at $1600^{\circ} \mathrm{C}$. The crystal structure was characterized by (XRD) and its morphology was examined by (SEM) and (TEM); the chemical composition was studied by (FTIR), (EDS) and (EELS), the texture characterization by (BET) and its potential in sunscreens by (FTIR and UV/VIS). Results have shown that boron nitride may be synthesized in the nanoscale and that this material has the potential to be incorporated into cosmetics.
\end{abstract}

Keywords: Boron Nitride; Nanostructure; Infrared Radiation; Cosmetics; Sunscreens

\section{Introduction}

The cosmetic industry currently has researched materials that offer advantages and which are attractive to customers. Ceramic materials are widely used in cosmetics and are manufactured by ceramic powder processing, sol-gel technology and precipitation solutions. The boron nitride is a ceramic material with a chemical formula (BN). Rhombohedral (r-BN), wurtzite (w-BN), cubic (c-BN) and hexagonal (h-BN) are the crystal structures of the $\mathrm{BN}$ caused by the arrangements of nitrogen and boron atoms $[1,2]$. The hexagonal structure allows the molecules to be arranged in layers so they slide one over the other and their use becomes interesting in cosmetic formulation to confer better lubricity mixtures.

Regarding the new researches in parameters used for the h-BN nanostructure synthesis aim to improve productivity, quality and control the size of synthesized particles. Recently, many studies have reported the preparation of nanostructures of the boron nitride with special morphologies, such as nanotubes [3,4], nanocapsules [5], nanocages [6] porous structures [7], hollow spheres [8], and nanofibers [9].

Traditionally, h-BN is prepared by the classical hightemperature synthesis routes, including a reaction of $\mathrm{B}_{2} \mathrm{O}_{3}$,

${ }^{*}$ Corresponding author. boric acid, or borax with carbon and nitrogen/ammonia and urea at a temperature around $2000^{\circ} \mathrm{C}$ [10]. On the other hand, for special $\mathrm{BN}$ nanostructures synthesis, laser ablation, chemical vapor-phase, carbothermal reduction of $\mathrm{B}_{2} \mathrm{O}_{3}$ and $\mathrm{B}_{4} \mathrm{C}$ and other methods have been developed [11]. Most of these methods cannot meet the needs of high yields, and therefore, the understanding on its synthesis is still a challenging subject. Moreover, BN nanotube obtained from different synthetic methods has different physical properties. Quality, quantity, and type of nanotubes synthesized depend on the synthetic method used.

In the cosmetics field, the particle size is important because smaller diameters may enable an increase in the surface area and the possibility of encapsulating cosmetics actives. The h-BN may offer advantages for use in cosmetics, particularly in sunscreens, since it allows manipulation of the structure during different phases of formation and, consequently, control of the size and the specific surface area; nevertheless, it has an excellent performance in the dispersion, it is non-toxic, transparent, and chemically inert.

Solar radiation is known to damage skin health and the cosmetic industry has invested in the research and manufacture of effective sunscreens. The range of the solar 
spectrum that normally reaches the surface of the Earth and has a special interest for photomedicine is that of ultraviolet B (UVB) and the Infrared (IR) [12]. The effect of ultraviolet rays is harmful to the health of the skin and can result in an increased cancer incidence, aging and other undesirable skin changes. Ultraviolet B is responsible for sunburn, erythema and inflammation. The effects of infrared rays are less described in the scientific literature, but these rays are known to increase the damages on the skin induced by ultraviolet rays [13], premature aging $[14,15]$ and infrared radiation may have a photocarcinogenic potential [16].

Sunscreens have been researched for a long time and currently they have been developed using the nanoscience and nanotechnology knowledge, as titanium dioxide, an important cosmetic used as sunscreen against ultraviolet radiations. However, for the protection against infrared radiation little has been researched. In the market, there are several inorganic actives such as titanium dioxide $\left(\mathrm{TiO}_{2}\right)$ and zinc oxide $(\mathrm{ZnO})$, which are able to block effects of ultraviolet radiation. These materials currently have smaller sizes and have advantages over the conventional product, as the phenomenon of radiation spread and therefore a white color under the skin [17].

With this regard, there is a great need to create formulations of sunscreens which can offer protection against ultraviolet rays as well as infrared rays. The boron nitride nanostructure is a potential material for this purpose as it is possible to manipulate its size, an important factor to use in sunscreens, thus reducing shine from oily skin as it has a high thermal conductivity. Indeed, it has absorption in the infrared ray region and special formulation containing titanium dioxide particles with nanostructured boron nitride would be a perfect combination to be applied as a cosmetic for protection against solar radiations in a broad spectrum.

\section{Experiments}

\subsection{Synthesis}

BN nanostructured samples were prepared using $12.36 \mathrm{~g}$ of boric acid $\left(\mathrm{H}_{3} \mathrm{BO}_{3}\right.$, Merck) dissolved in $400 \mathrm{ml}$ of distilled water at $100^{\circ} \mathrm{C}$. After dissolution $12.60 \mathrm{~g}$ of melamine $\left(\mathrm{C}_{3} \mathrm{H}_{6} \mathrm{~N}_{6}\right.$, Merck) was slowly added to the medium under agitation. We observed the formation of a precipitate early in the reaction. The material was allowed to stand for 48 hours at room temperature. Then it was filtered and dried at $37^{\circ} \mathrm{C}$. The product obtained is $\mathrm{B}_{4} \mathrm{~N}_{3} \mathrm{O}_{2} \mathrm{H}$, which is the precursor to $\mathrm{BN}$. This precursor material was heated in tubular furnace without gas flow at $500^{\circ} \mathrm{C}$ for 3 hours constantly and followed by nitrogen gas flow, for 1 hour at $800^{\circ} \mathrm{C}\left(5^{\circ} \mathrm{C} / \mathrm{min}\right)$ at a constant temperature. This precursor was placed in an aluminum boat and heated at $1600^{\circ} \mathrm{C}\left(10^{\circ} \mathrm{C} / \mathrm{min}\right)$ for 2 hours with nitrogen gas flow.

\subsection{Characterization}

The prepared samples were characterized by Fouriertransform infrared spectroscopy (FTIR), X-ray diffraction (XRD), Brunauer-Emmett Teller (BET), scanning electron microscopy (SEM), energy dispersive spectroscopy (EDS), transmission electron microscopy (TEM) and Spectroscopy (UV/VIS). FTIR measurements were carried out in a Galaxy-Matson 3020 spectrophotometer ranging from 4000 to $400 \mathrm{~cm}^{-1}$ at room temperature using $\mathrm{KBr}$ pellets. XRD patterns were obtained using a Rigaku Geigerflex diffractometer with a $\mathrm{Cu}-\mathrm{Ka}$ tube. The specific surface area was measured by Quantachrome NOVA 1200 adsorption analyzer. SEM characterization was performed in a scanning electron microscope (JEOL, JSM, 840) operating at $15 \mathrm{kV}$. EDS was performed by (JEOL JXA-8900R).TEM characterization was performed in a transmission electron microscope (JEOL, JEM, $2100 \mathrm{~F}$ ) with an acceleration potential of $200 \mathrm{kV}$. UV/VIS characterization was performed in a Shimadzu model (2401) spectrometry.

\section{Results and Discussion}

The synthesis begins with the formation of the melamine diborate precursor [18]. It was prepared from boric acid and melanin reaction as below. They can be described in Equation (1) (see below).

Various temperatures and heating rates were tested after preparing the precursor and which showed the best results when the sample was subjected to $1600^{\circ} \mathrm{C}$ at a heating rate of $10^{\circ} \mathrm{C} / \mathrm{min}$ for 2 hours with $\mathrm{N}_{2}$. For this synthesis, an initial 2-hour purge was carried out in order to minimize the presence of contaminants. This was crucial to the proposed result.

Figure 1 shows the FTIR spectra of samples treated at $1600^{\circ} \mathrm{C}$. The sample has characteristic peaks in the hexagonal boron nitride formation. The spectra showed an asymmetric bond at $1380 \mathrm{~cm}^{-1}$ which corresponds to B-N stretch and the bond at $775 \mathrm{~cm}^{-1}$ assigned to the connection which may be attributed to B-N-B. The band at 3400 $\mathrm{cm}^{-1}$ can be attributed to the moisture absorbed. The spectrum has to be free of impurities, which are related to other crystalline forms of boron nitride. It is noteworthy

$$
2\left(\mathrm{C}_{3} \mathrm{~N}_{6} \mathrm{H}_{6} \cdot 2 \mathrm{H}_{3} \mathrm{BO}_{3}\right) \rightarrow \mathrm{B}_{4} \mathrm{~N}_{3} \mathrm{O}_{2} \mathrm{H}+4 \mathrm{H}_{2} \mathrm{NCN}+2 \mathrm{CO}_{2}+\mathrm{NH}_{3}+6 \mathrm{H}_{2} \mathrm{O}
$$


that this technique generates important data to characterize the h-BN phase, since it is possible to distinguish $\mathrm{sp}^{2}$ bonds of hexagonal h-BN phase samples and $\mathrm{sp}^{3}$ of cubic c-BN phase. According to Hao and colleagues [19] $\mathrm{sp}^{2}-$ type bonds (h-BN) are thermodynamically stable under the synthesis conditions of this work, whereas for the formation of $\mathrm{sp}^{3}$ bonds typical of c-BN, there is a kinetics barrier to formation. Other authors have presented similar results in which the formation of h-BN occurs at around $1380^{\circ} \mathrm{C}[20,21]$. Peaks in 1096 and $1166 \mathrm{~cm}^{-1}$ are due to the formation of c-BN phase and they were not found in the sample treated at $1600^{\circ} \mathrm{C}$.

Figure 2 shows the $\mathrm{X}$-Ray diffraction pattern of the sample treated at $1600^{\circ} \mathrm{C}$. The peaks at $2 \theta=26.32^{\circ}, 2 \theta=$ $41.60^{\circ}$ and $2 \theta=55.00^{\circ}$ (JPCD, No. 9 - 12) may be attributed to hexagonal boron nitride formation. The peak at $26^{\circ}$ corresponds to the (002) diffraction peak of h-BN [19] and it is very intense and well defined. This sample is more crystalline compared to other temperatures tested. The turbostratic structure (imperfect structure with no three-tridimensional order) has been produced at a low temperature. This structure is transformed in an ordered hexagonal from by heat treatment at a temperature above $1500^{\circ} \mathrm{C}[22]$.

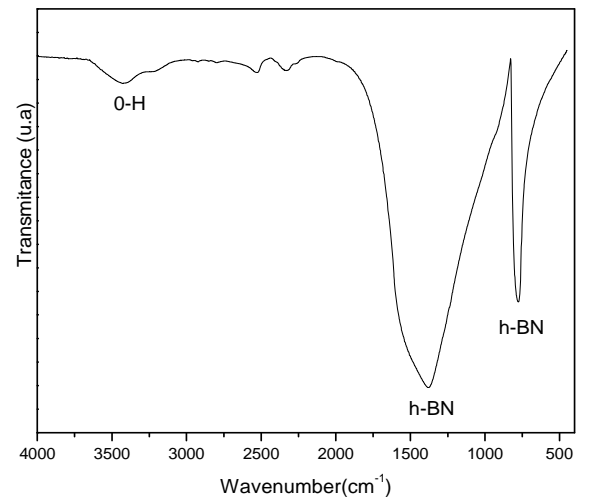

(a)

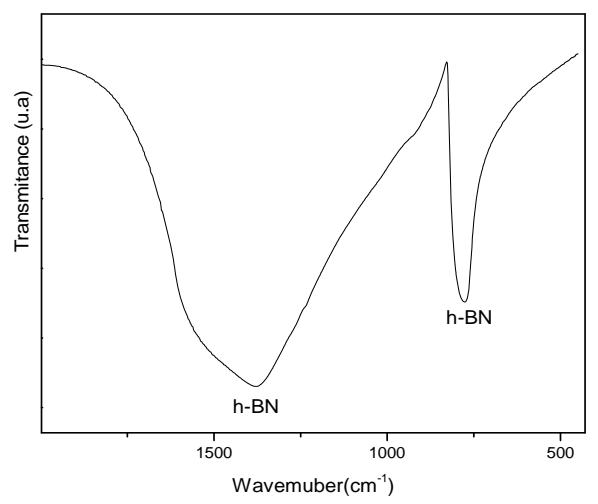

(b)

Figure 1. (a) Infrared spectrum of the sample treated at $1600^{\circ} \mathrm{C}$, and (b) Expanded Infrared spectrum treated at $1600^{\circ} \mathrm{C}$.

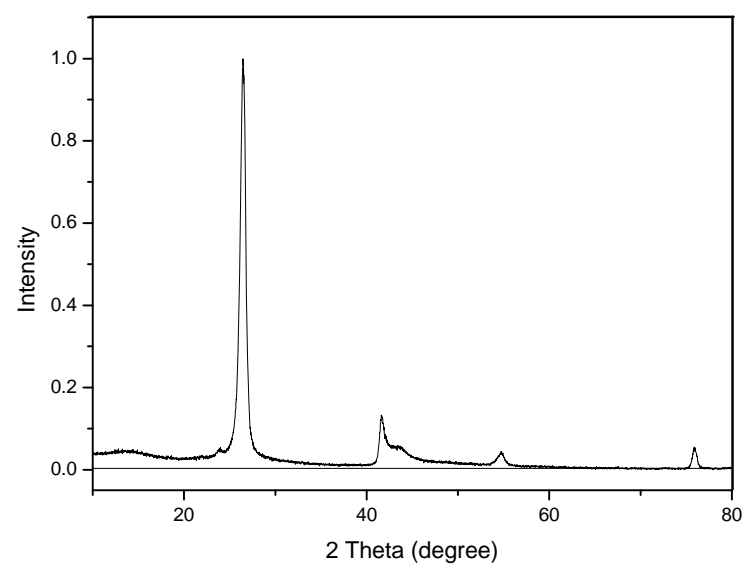

Figure 2. XRD pattern of the sample treated at $1600^{\circ} \mathrm{C}$.

From the diffraction data, we measured the particle size according to the Scherrer equation:

$$
D_{h k l}=K l / b_{h k l} \cos q_{h k l},
$$

where $D_{h k l}$ is the particle size perpendicular to the normal line of $(h k l)$ plane, $\mathrm{k}$ is a constant (it is about 0.9$), \beta_{h k l}$ is the full width at half height of the ( $h k l)$ diffraction peak, $\theta_{h k l}$ is the Bragg angle of $(h k l)$ peak and $\lambda$ is wavelength of X-ray. The average size of the particle found was 15 $\mathrm{nm}$. This value is important for the objective of the study since it shows that the material is in the nanoscale.

Figure 3 shows nitrogen isotherm and pore distribution of the sample. According to the literature, boron nitride may have a surface area between $\left(5 \mathrm{~m}^{2} / \mathrm{g}-820\right.$ $\mathrm{m}^{2} / \mathrm{g}$ ) but these values depend on factors related to the synthesis conditions and polymeric precursors used [23]. The value found $\left(90 \mathrm{~m}^{2} / \mathrm{g}\right)$ may be considered interesting since the increasing temperature leads to a decrease in the surface area. This material also has the potential to be used as a matrix for embedding drugs due to area surface. The isotherm is similar to type IV (mesoporous material) with micropores contribution and display type $\mathrm{H} 3$ hysteresis loop according to the IUPAC classification, which is often observed with aggregates of plate-like particles that give rise to slit-shaped pores. The adsorption of the gas first starts with micropores and then continues with mesopores. The adsorption and desorption mechanism occurs at the same time in micropores. While adsorption occurs by the condensation towards the walls of pores, desorption occurs by evaporation from the walls of pores. In mesopores, adsorption and desorption follow different ways. The filling of the mesopores occurs by the condensation on the wall of pores with formation of layers. On the other hand, desorption occurs by moving away from pore opening with the evaporation. Hence, in mesoporous region where the $\mathrm{P} / \mathrm{Po}>0.4$, type $\mathrm{H} 3$ hystreresis was observed. This experiment revealed that the final material possess a narrow pore size distribution with an average pore size of about $3.6 \mathrm{~nm}$, characteristic of 
mesoporous materials. The pore size distribution (Figure 3(a)) for the produced nanostructured boron nitride was plotted using desorption data of BJH (Barrett-JoynerHalenda) method at $0.35<\mathrm{P} / \mathrm{Po}<0.99$ corresponding to mesoporous site. The material presents a total pore volume of $0.26 \mathrm{cc} / \mathrm{g}$ calculated using the BJH method and with a pore size of $3.6 \mathrm{~nm}$ in diameter. Table 1 shows the main results obtained from the $\mathrm{N}_{2}$ adsorption.

Figure 4 shows the micrograph of the sample treated at $1600^{\circ} \mathrm{C}$. The formation of an agglomerate of fine grain of hexagonal boron nitride with diameter around $0.5 \mu \mathrm{m}$ is observed. This image is important to demonstrate the application of the material studied in the field of cosme-

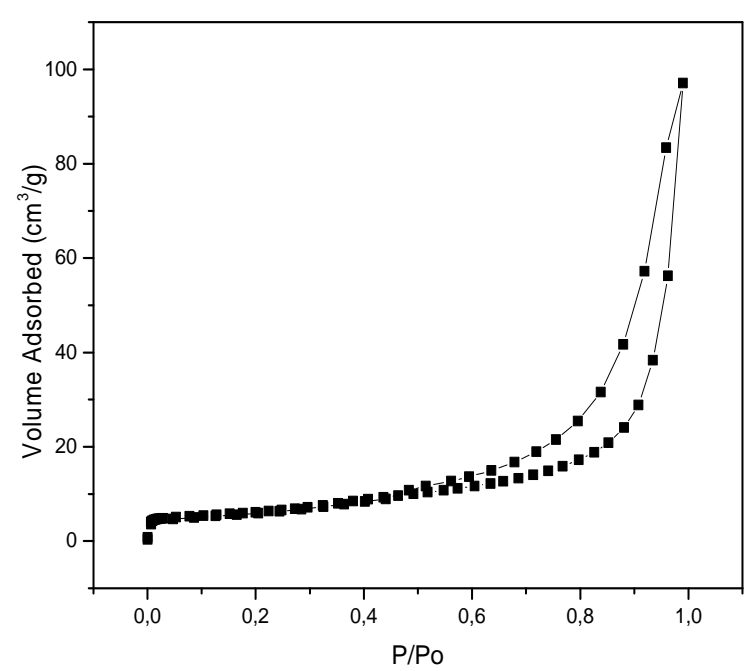

(a)

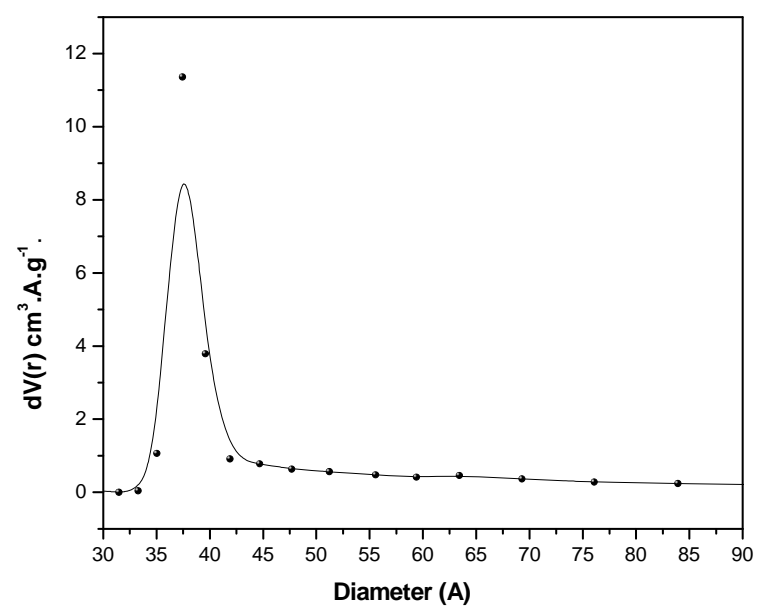

(b)

Figure 3. (a) Isotherms of Nitrogen absorptions and (b) pore distribution of the sample treated at $1600^{\circ} \mathrm{C}$.

Table 1. Nitrogen Adsorption Results.

\begin{tabular}{rccc}
\hline Sample & $\mathbf{S}\left(\mathbf{m}^{2}\right)$ & $\mathbf{V p}(\mathbf{c c} / \mathbf{g})$ & $\mathbf{D p}(\mathbf{n m})$ \\
$1600{ }^{\circ} \mathrm{C}$ & 90 & 0.26 & 3.6 \\
\hline
\end{tabular}

tology. Others researches have reported a similar morphology at temperatures above $1600^{\circ} \mathrm{C}$.

Figure 5 shows a typical EDS boron nitride. This figure illustrates the general analysis of the material surface. There are only boron and nitrogen atoms. The results allow us to verify the homogeneity of the sample containing the boron and nitrogen. This result was obtained due to the purge of the sample before conducting the synthesis with nitrogen gas flow for two hours. This procedure was important to minimize the presence of contaminants in the sample.

Figure 6 shows TEM image of the boron nitride obtained at $1600^{\circ} \mathrm{C}$. The image confirms nanoscale of the synthesized samples and the formation of primary particles of fine grains. The microstructure has approximately $0.5 \mu \mathrm{m}$ in diameter and $5 \mu \mathrm{m}$ in length. The high-resolution transmission electron microscopy image shows a spacing layer of about $0.33 \mathrm{~nm}$, corresponding to the interplanar distance (002). This finding is in agreement with values reported for interplanar distance of h-BN presented in the literature [18].

Figure 7 shows the spectrum of the samples at $1600^{\circ} \mathrm{C}$. Two different absorptions have been observed, near 188 $\mathrm{eV}$ and $401 \mathrm{eV}$ as described in the literature [18] being assigned to the hexagonal boron nitride. These figures refer to boron and nitrogen, respectively. The quantification of this analysis shows a ratio close to $1: 1$, thus,

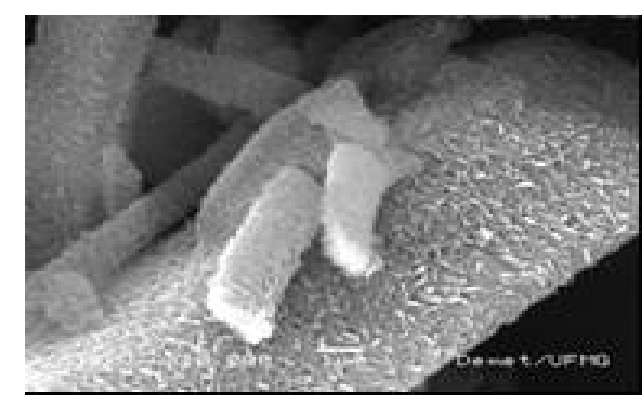

Figure 4. SEM micrograph of a sample treated at $1600^{\circ} \mathrm{C}$.

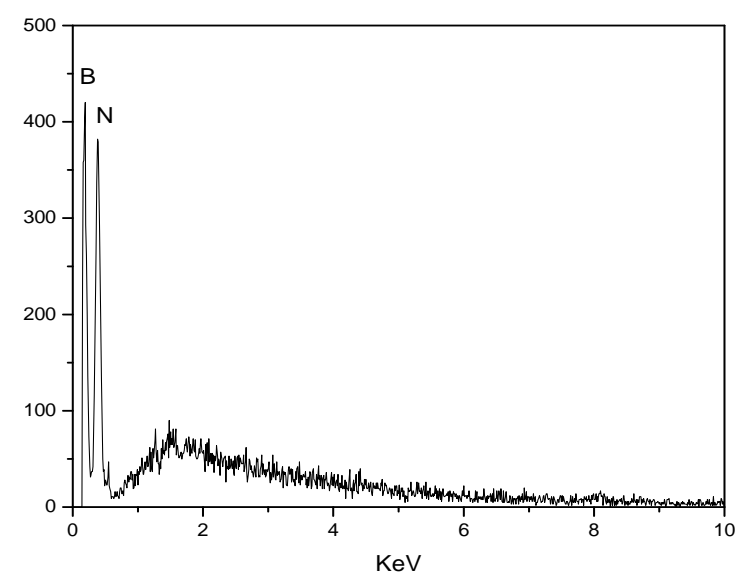

Figure 5. EDS spectrum of the sample treated at $1600^{\circ} \mathrm{C}$. 


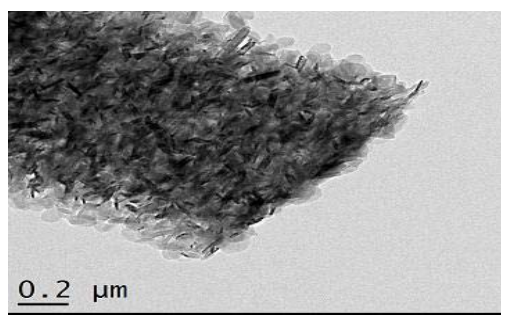

(a)

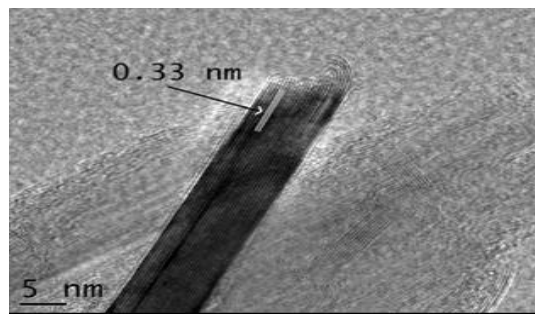

(b)

Figure 6. (a) TEM; (b) HRMET image of sample treated at $1600^{\circ} \mathrm{C}$.

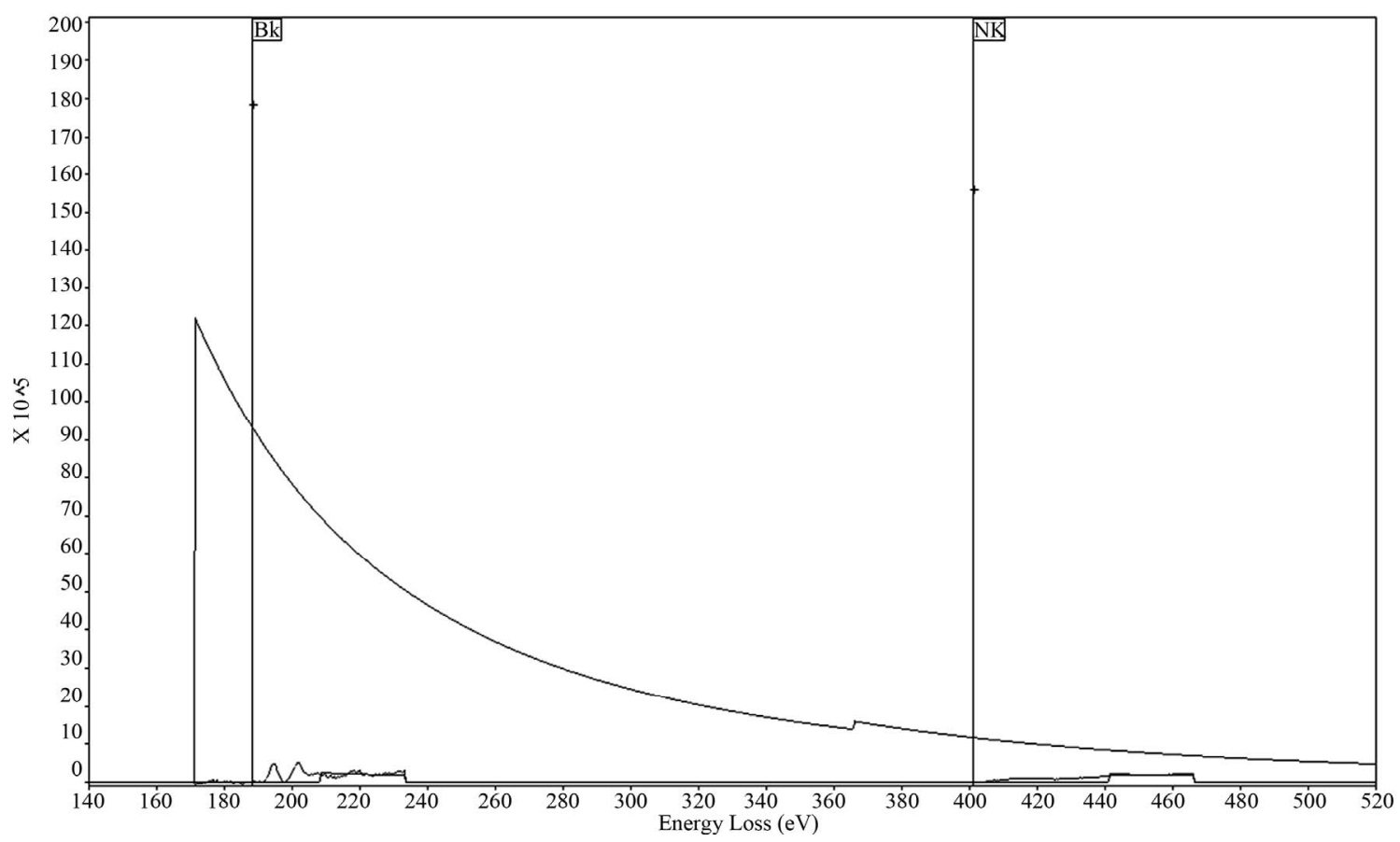

Figure 7. Spectrum EELS of the sample treated at $1600^{\circ} \mathrm{C}$.

showing the formation of pure boron nitride (BN). Interestingly, no other element has been detected in this sample.

Figure 8 shows the crystallography of the sample at $1600^{\circ} \mathrm{C}$ with several diffraction rings. These rings can be indexed as (002), (100), (004) and (110) reflections, which are typical of hexagonal boron nitride. SAED analysis shows correspondence of the reflections with those of BN JCPDS \# 34-0421 and this result is also confirmed by XRD, as discussed.

Figure 9 shows the FTIR comparison between the sample obtained at $1600^{\circ} \mathrm{C}$ and titanium dioxide $\left(\mathrm{TiO}_{2}\right)$. The spectrum of infrared radiation may be divided according to three sub-regions: IR-A $(760 \mathrm{~nm}-1400 \mathrm{~nm})$, IR-B $(1400-3000 \mathrm{~nm})$ and IR-C (3000 nm - 1mm). Other possibility is: near -IR $(760 \mathrm{~nm}-3000 \mathrm{~nm})$, medium IR-B (3000 - 30,000 nm) and far IR (30,000 nm - 1 $\mathrm{mm})$. The absorption of infrared radiation is different in various layers of the skin and infrared radiation penetrates more deeply into the skin when compared to the ultraviolet radiation [16]. Depending on the penetration into the skin, it is possible occur damage in cellular responses, a heating sensation and burning takes place. Thus, it is of great importance to consider this characteristic, since these materials have the ability to block effects of hard radiation and, consequently, to minimize such reactive effects in the human health. Titanium dioxide has an absorption band different from that of boron nitride. The region of the spectrum boron nitride is wider than the titanium dioxide which indicates an additional photoprotection when it is associated with a cosmetic formulation. As previously mentioned, this photoprotection is important to minimize the damage in the skin.

Figure 10 shows the UV-VIS comparison of the sample obtained at $1600^{\circ} \mathrm{C}$ and titanium dioxide $\left(\mathrm{TiO}_{2}\right)$. The boron nitride has a high reflectivity in the visible spectrum and thus, its properties in this wavelength are insignificant. Thus it is not indicated for use in photoprotection in the UV/VIS range alone. Titanium dioxide is more effective than boron nitride hexagonal in this range 


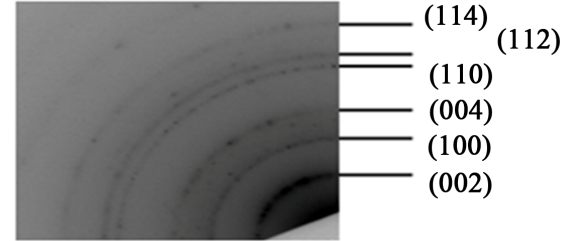

Figure 8. SAED patterns of the sample treated at $1600^{\circ} \mathrm{C}$.

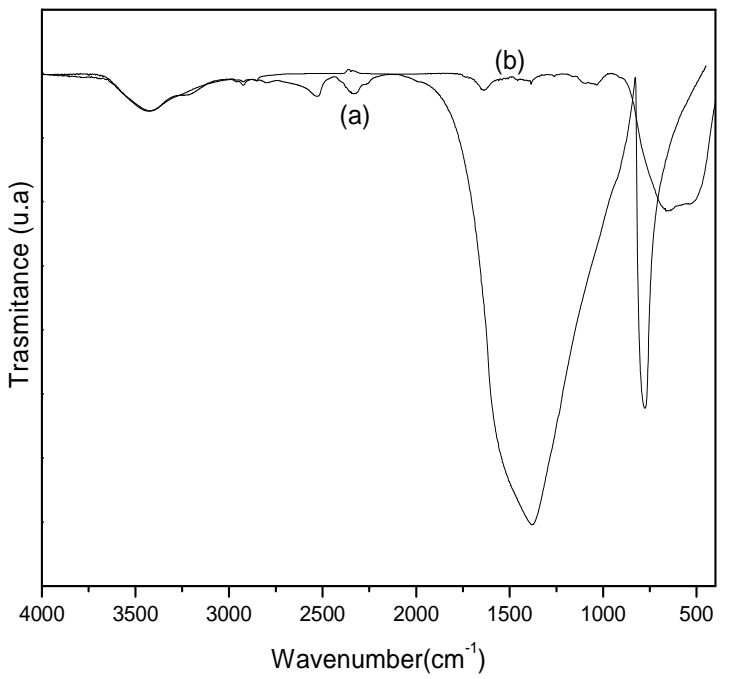

Figure 9. (a) Infrared spectrum of the sample (a) treated at $1600^{\circ} \mathrm{C}$ and (b) $\mathrm{TiO}_{2}$.

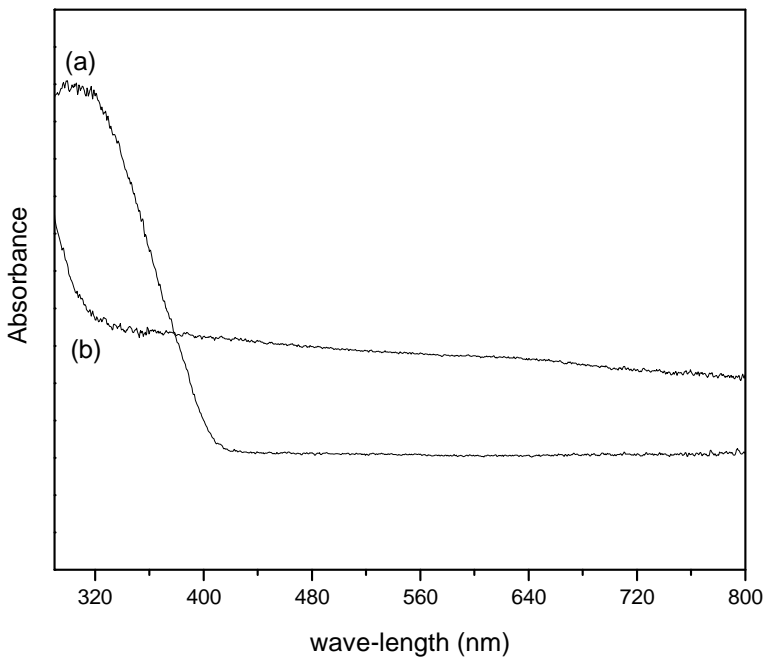

Figure 10. Spectrum UV/VIS (a) $\mathrm{TiO}_{2}$ and (b) sample treated at $1600^{\circ} \mathrm{C}$.

of radiation. Depending on the particle size, the protecttion of the titanium dioxide may occur not only through reflection but also through absorption.

\section{Conclusion}

The material developed in this synthesis provides chemical and structural features similar to those described in the literature for the formation of hexagonal boron nitride. The heat treatment at $1600^{\circ}$ was critical to the result obtained, since it led to the formation of hexagonal nanostructure. The particle size is found in the order of nanometers, being important result for the proposed theme. The ability to absorb in the infrared range makes these materials a powerful adjuvants together titanium dioxide for application in sunscreens.

\section{Acknowledgements}

This study has been supported by CAPES, CNPq and FAPEMIG. The authors thank the Microscopy Center of UFMG for Microscopy Images.

\section{REFERENCES}

[1] J. Y. Huang and Y. T. Zhu, "Advances in the Synthesis and Characterization of Boron Nitride," Defect and Diffusion Forum, Vol. 186-187, No. 1, 2000, pp. 1-32.

[2] W. J. Yu, W. M. Lau, S. P. Chan, Z. F. Liu and Q. Q. Zheng, "Ab Initio Study of Phase Transformations in Boron Nitride," Physical Review B, Vol. 67, No. 1, 2003, pp. 14108-14116. doi:10.1103/PhysRevB.67.014108

[3] Y. Chen, M. Conway, J. S. Williams and J. Zou, "LargeQuantity Production of High-Yield Boron Nitride Nanotubes", Journal of Materials Research, Vol. 17, No. 8, 2002, pp. 1896-1899. doi:10.1557/JMR.2002.0281

[4] C. Zhi, Y. Bando, C. Tang and D. Golberg, "Boron Nitride Nanotubes," Materials Science and Engineering $R$, Vol. 70, No. 3-6, 2010, pp. 92-111.

doi:10.1016/j.mser.2010.06.004

[5] S. Komatsu, Y. Shimizu, Y. Moriyoshi, K. Okada and M. Mitomo, "Preparation of Boron Nitride Nanocapsules by Plasma Assisted Pulsed Laser Deposition," Journal of Applied Physics, Vol. 91, No. 9, 2002, pp. 6181-6184. doi:10.1063/1.1461889

[6] T. Oku, M. Kuno and H. Kitahara, "Formation, Atomic Structures and Properties of Boron Nitride and Carbon Nanocage Fullerene Materials," Internacional Journal of Inorganic Materials, Vol. 3, No. 7, 2001, pp. 597-612. doi:10.1016/S1466-6049(01)00169-6

[7] P. Dibandjo, L. Bois, F. Chassagneux and P. Miele, "Thermal Stability of Mesoporous Boron Nitride Template with a Cationic Surfactant," Journal of the European Ceramic Society, Vol. 27, No. 1, 2007, pp. 313-317. doi:10.1016/j.jeurceramsoc.2006.04.178

[8] L. Y. Chen, Y. L. Gu, L. Shi, Z. H. Yang, J. H. Ma and Y. T. Qian, "A Room-Temperature Approach to Boron Nitride Hollow Spheres," Solid State Communication, Vol. 130, No. 8, 2004, pp. 537-540. doi:10.1016/j.ssc.2004.03.009

[9] H. J. Hwang, N. A. M. Barakat, M. A. Kanjwal, F. A. Sheikh and H. Y. Kim, "Boron Nitride Nonofibers by the Electrospinning Tecnique," Macromolecular Research, Vol. 18, No. 6, 2010, pp. 551-557. doi:10.1007/s13233-010-0601-2 
[10] R. S. Kalyoncu, "BN Powder Synthesis at Low Temperatures," Ceramic Engineering and Science Proceedings, Vol. 6, No. 9-10, 1985, pp. 1356-1363. doi:10.1002/9780470320297.ch18

[11] L. Gao and J. Li, "Preparation of Nanostructured Hexagonal Boron Nitride Powder," Journal of American Ceramic Society, Vol. 86, No. 11, 2003, pp. 1982-1984. doi:10.1111/j.1151-2916.2003.tb03596.x

[12] J. E Costa and E. Lascaz, "Fotoprotetores," Medicina Cutánea Ibero-Latino Americana A, Vol. 29, No. 3, 2001, pp. 145-152.

[13] L. H. Kligman, "Intensification of Ultraviolet-Induced Dermal Damage by Infrared Radiation," Archives of Dermatological Research, Vol. 272, No. 3-4, 1982, pp. 229238. doi:10.1007/BF00509050

[14] S. Cho, M. H. Shin, Y. K. Kim, J. E. Seo, Y. M. Lee, C. H. Park and J. H. Chung, "Effects of Infrared Radiation and Heat on Human Skin Aging in Vivo," Journal of Investigative Dermatology Symposium Proceedings, Vol. 14, No. 1, 2009, pp. 15-19. doi:10.1038/jidsymp.2009.7

[15] C. Calles, M. Schneider, F. Macaluso, T. Benesova, J. Krutmann and P. Schroeder, "Infrared A Radiation Influences the Skin Fibroblast Transcriptome: Mechanisms and Consequences," Journal of Investigative Dermatology, Vol. 130, No. 6, 2010, pp. 1524-1536. doi:10.1038/jid.2010.9

[16] S. M. Schieke, P. Schroeder and J. Krutmann, "Cutaneous Effects of Infrared Radiation: From Clinical Observations to Molecular Response Mechanisms," Photodermatology Photoimunology \& Photomedicine, Vol. 19, No. 5, 2003, pp. 228-234. doi:10.1034/j.1600-0781.2003.00054.x
[17] J. Flor, M. R. Davolos and M. A. Correa, "Protetores Solares," Química Nova, Vol. 30, No. 1, 2007, pp. 153-158. doi:10.1590/S0100-40422007000100027

[18] R. Ma, Y. Bando and T. Sato, "CVD Synthesis of Boron Nitride Nanotubes without Metal Catalysts," Chemical Physics Letters, Vol. 337, No. 1-3, 2001, pp. 61-64. doi:10.1016/S0009-2614(01)00194-4

[19] X. Hao, M. Yub, Z. Cui, X. Xu, Q. Wang and M. Jiang, "The Effect of Temperature on the Synthesis of BN Nanoscrystals," Journal of Crystal Growth, Vol. 241 No. 1-2, 2002, pp. 124-128. doi:10.1016/S0022-0248(02)01291-5

[20] J. Vilcarromero, M. N. P. Carreño and I. Pereyra, "Mechanical Properties of Boron Nitride Thin film Obtained by RF-PECVD at Low Temperatures," Thin Solid Films, Vol. 373, No. 1-2, 2000, pp. 273-276. doi:10.1016/S0040-6090(00)01096-8

[21] S. Y. Xie, W. Wang, K. A. Shiral-Fernando, X. Wang, Y. Lin and Y.-P. Sun, "Solubilization of Boron Nitride Nanotubes," Chemical Communications, No. 29, 2005, pp. 3670-3672. doi:10.1039/b505330g

[22] T. Hagio, K. Nonaka and T. Sato, "Microstructural Development with Crystallization of Hexagonal Boron Nitride," Journal of Materials Science Letters, Vol. 16, No. 10, 1997, pp. 795-798. doi:10.1023/A:1018518222594

[23] C. K. Narula, R. Schaeffer, A. K. Datye, T. T. Borek, B. M. Rapko and R. T. Paine, "Synthesis of Boron-Nitride Ceramics From Oligomeric Precursors Derived From 2-(Dimethylamino)-4,6-dichloroborazine," Chemistry of Materials, Vol. 2, No. 4, 1990, pp. 384-389. doi: $10.1021 / \mathrm{cm} 00010 \mathrm{a} 014$ 